\title{
The Investigation of the Nature's Role on the Life Quality of the Elderly Toward Promoting Interactions and Social Sustainability
}

\author{
Karimeh Silave $^{1} \quad$ Mohamad Shikh Bahaee ${ }^{2 *}$ \\ Islamic Azad University Naein Branch, Naein, Iran \\ Islamic Azad University, Khomeyni Shahr Branch, Khomeyni Shahr, Iran
}

\begin{abstract}
Paying attention to human needs in every individual's health cycle results in the healthy community. There is a group of people who are in need of support which are called elderly community. In architecture, not only the principles and standards must be defined, established and observed, but also should defined a certain specific spatial pattern to these individuals. Social sustainability in the broad sense, is synonymous with raising the quality of human life of the present generation and future generation, considering his or her talents and capacities and satisfying the needs of all classes. So, there is an indicator such as increased level of education, social justice and popular participation, etc. The architectural and urban environment is one of the most important elements in relation to the elderly's life. In order to maximize the benefits of the elderly in services and society, elderly life and their spirituality must be carefully analyzed. The present article addresses the needs of the elderly from a social dimension to the public space in a container for social interaction, and seeks to step up awareness and understanding of the perception of nature in order to design an appropriate space architecture for the social interaction of the elderly in their own housing spaces. The applied methodology for the present research is descriptive-analytic, and data collection is library method.
\end{abstract}

Keywords: Elderly, social interactions, social sustainability, nature

DOI: $10.7176 / \mathrm{ADS} / 73-02$

Publication date:May $31^{\text {st }} 2019$

\section{Introduction}

Aging phenomenon occurs due to natural series of event in which physiological and psycho social changes happen in the body. Generally speaking, aging is an emerging period with a number of specific physical and psychological limitations that, without knowing the nature and quality of these limitations, it will not be possible to consider appropriate environment to elderly people. If the main goal of environmental adaptation for the elderly is to provide facilities to compensate for their physical and psychological limitations and to maximize the efficacy of limited capabilities, knowing the limitations of the elderly and their negative impact is the most important and fundamental role in this field (Rafizadeh, 2003; p. 112).

Aging is one of the crucial and decisive stages of human development, which is, contrary to common belief, it is not only the end of life, but also the natural process of passing through life. Aging is a personal and social experience. This might be recognized as a pleasure for some people, and for others it's sad and boring process (Pourjafar, Taghahi, Bemanian, Sadeghi and Ahmadi, 2010; 22).

People within all ages meet their emotional needs, spiritual needs, special talents of that age. Elderly people, after retirement, instead of enjoying and relaxing, people develop mental depression due to inefficiency and rejection, and this has a great impact on their useful life and the loss of life expectancy. Such people spend their time to share either alone or with people who are not mentally in a better position because of their lack of proper social companionship. Due to the attention to the human needs at each level individual health, it is necessary to take needs of the people into consideration.

Many elderly people describe aging as a monotone and fearful, as it is an unpleasant experience (1961, Moustakas, Heravi Karimavi, Rejeh, Jalali, Pishnamazi, 2003; p.p, 59-66). Evidence suggests that loneliness is a widespread phenomenon, affecting $22-25 \%$ of the total population over the age of 52 in terms of age and sex (Shearer R, Davidhizar, 1999; p.p, 12-15).

Long et al. (1998) in the longitudinal and cross sectional studies stated that the size of the social network and the amount of social interaction were reduced in almost all elderly people. According to separate studies, Long and Balts (1997), Adukins, Martin and Powon (1996) stated that social contact and peoples personality are related to the satisfaction of everyday life and high morale in old age (Pasha; Safarzadeh; Mashak, 2007; p. 506). In this regard, in the field of architecture, efforts to create public spaces adapted to the physical and mental conditions of the elderly seem effective and can be effective in improving their social interactions and preventing social isolation. Social interaction is more than any other activity to the sensitive environment. Human activities in the context of a complex system, which have different aspects and different theoretical approaches. These approaches share a common conception of the concept of quality of life in the human environment, which reveals the need to address the capabilities of the human environment. The possibility of establishing favorable collective interactions at different levels of collective creation in the human environment, on the one hand, and 
the possibility of perception of nature and natural elements, on the other, implies the two aspects of the qualities of the human environment, which have been explored in this research. The interest in nature and the desire to be close to the natural elements of life are the feelings that are widely used by users of the built environment, and this affects the quality of life in the places of activity.

\section{Elderly}

In the Iranian society, ages 65 to 70 have begun aging (Rafizadeh, 2001; 17). But you cannot be aged for a specific age. Because when some people reach 45, while others over 65 age, adapt themselves to age-related changes and maintain their relationship with family and community (Lencaroni, 1999). Aging is a kind of weakness in mental and physical forces that occur over time. Aging definition in different countries varies according to cultural conditions. In other words, an old age does not exist in the real sense, the feeling of aging is an internal and mental issue that varies from person to person. During this period, the elderly can be classified as follows:

1. Aged people who can think but lose their physical strength.

2. Aged people who have lost their physical and intellectual powers.

3. Aged people with active body and mind (Shamloo, 1985)

The needs of the elderly can be summarized in the following three groups

1. Emotional needs

2. Livelihood needs

3. Need to have fun (Stewarat, 1998)

4. Need to participate in social life

5. Security (Daryadar, 2007)

\section{Social sustainability}

In sustainable design, social sustainability must be taken into account in terms of energy consumption and the environmental impact of buildings and cities. Despite numerous studies on environmental sustainability and reducing energy consumption in buildings, it can be said that the sustainability dimension in design has so far been largely neglected. In recent years, there has been a prerequisite for the sustainability of social factors. The main axis of social sustainability of man is the present and future generations (Tabatabaei and Aminzadeh, 2013; p. 3). Sustainable social development can be considered as a general positive dimension in design, and has been largely neglected. In recent years, there has been a prerequisite for the sustainability of social factors. The main axis of social sustainability of man is the present and future generations (Tabatabaei \& Aminzadeh, 2013; p. 3). Chapman stated that the goal of this development, which is based on justice and stability, is to maintain the quality of human life in terms of the capacity of the planet. He says that there are forms of development that increase freedom and mobility, and that other types may affect people's attitudes (Chapman , 2005, p. 124).

Sustainable social development means radical transformations in social structures and relationships with the motivation to realize the goals of social integration, social cohesion, meeting needs along with raising the level of freedom and enhancing community's ability to improve the state of self-improvement of the vitality of civil society and increasing social and cultural values of change. The development of the social system is aimed at increasing the quality of life of all members of society by taking into account the talents and capacities of the society (Bazrafkan \& Ghakkub, 2011; p. 2).

3-1. Measures for the development of social sustainability in architecture

Regarding the principles of social sustainability and its indices and criteria in society, and by reviewing the research carried out in other fields, the indicator of the development of social sustainability in architecture are as follows:

1. Social interaction

In the direction of the development of social sustainability, one of the things that should be considered architecturally more than before is the discussion of social interaction in architecture. The human need for social interaction at different levels is not covered by anyone, and the physical space design for this interaction is the task of architect designers. The architectural space's social capability can reduce or increase the amount of our social interaction among people in space, and consequently increase social capital and, consequently, the development of social sustainability.

2. Architectural identity

Despite its territorial differences, architecture has inherent dynamic and gradual features that call it identity (Taqvaei, 2012; p. 65). The design of spaces with identity rooted in the culture and history of the community is unique to the architecture of any land. Creating spaces with architectural identity can increase the sense of social identity and reduce this feeling compared to the design of imported architecture that is rooted in culture and history unique to a land (Davoodi, Fallah \& Ali Abadi, 2013; p. 6 )

3. Social Security 
Another indicator for developing social sustainability in architecture is sense creation of security in space. The design of architectural space in such a way as to increase the sense of controllability in users, the design of safe urban spaces, and the design of a defensible space, etc., are the architectural considerations that must be considered. Therefore, consideration of the security architecture issue for the development of social sustainability is essential. It should be noted that there are other indicators such as comfort, social order, flexibility, etc., which in turn are important to the indicators presented (ibid, 7).

4. Social activities

Social interaction means the reaction of a relationship between two or more people that leads to an interaction between them, and this kind of reaction is recognizable to both parties. Therefore, meaningless relationships do not fall within this definition. Of course, there are other definitions for social interactions. For example, social interaction and communication can be a physical issue, a look, a conversation, and a connection between individuals, which itself requires the definition of events and activities that are proportional and, consequently, the role of people in Space and their membership in social groups and networks (Daneshpour \& Charkhchian, 2007; p. 22).

The social system consists of a group of individuals who, directly or indirectly, have a regular social relation for a particular purpose. Each social system requires human communication for the study of norms, the determination of the roles and the allocation of each person's personality, the level of responsibility, and the position of the people towards each other for survival. In other words, in order to continue the life of human social life and the growth of the culture of human communities, we are bound to communicate verbally and faceto-face, and these happen in the built-in spaces of the physical form and become objective. The desired level of social interaction depends on personal characteristics, social influences, physical environment and culture (Gifford, 1997).

Social interactions are unofficial and unpredictable, that is, without prior appointments in public spaces, as well as the opportunity to meet people can be by taking into account a series of lateral spaces and support such as service spaces, commercial and recreational spaces. Increased public space. Urban designers and designers design the environment to provide opportunities for social relationships (Skjaeveland, 1997).

5. Human`s social needs

Addressing human needs is very important that researchers in various disciplines, including social sciences, have defined the ultimate goal of social development to meet human needs. From the perspective of this researcher, the Maslow Human Hierarchy can be classified into three categories:

1. Biological and physiological needs

2. Psychological Needs

3. Social Needs (Seyfollahi, 2002).

According to this view, social needs (the need to foster collective efforts in solving problems, the need for empathy) is one of the most basic needs that every human being depends on to survive. Therefore, human being is a social being and social interaction and communication is one of his basic needs. Often, human beings interact with others who know their abilities, create meaning and make their own identity (Thivud, 2000). From Christian Christopher Alexander's perspective, social interaction is essential to respond to human needs by connecting and feeling belonging to a place. He also believes that activities such as interacting with others and observing the activities of the people, helping to create the grounds for socialization and socialization contribute to individual human development. At the same time, social interaction approaches each other's attitudes toward mental backgrounds and characteristics (Alexander, 2008).

6. Elderly Need for Social Interactions

Social interactions are considered as important in the health and well-being of the elderly and are created through direct communication with the public. Social interactions in reducing mortality, improving the quality of life, reducing symptoms of depression and reducing cognitive impairment are influential (Seddiysi, Dashemjir \& Dehdar, 1391; p. 50). Research has shown that elderly people who are present in social activities despite being older, Have relatively good social relations, suffer from a variety of illnesses that are less likely to develop mental illness (Hashemzadeh \& Asadi Boalourdi, 2009; p. 82).

6 . The public space, the context of social interactions

Social interaction is more sensitive to the environment than any other activity. The physical environment of interaction is what Joseph P. Fergus calls "boom". He believes that most people are unaware of the effects of the physical environment in their encounters. He points to two aspects of the architectural environment and their impact on the social interaction of individuals in space.

1. Static aspect of architectural space

2. The Dynamic Aspect of Architectural Space (Fergus, 2000)

The pattern of social interaction and the capabilities of the architectural space made for this reason make it important that there is a close relationship between social interaction and the attachment of people to social and constructive environments (Long, 2002). The link between architectural space and social interaction is not 
straightforward, and the way cognitive perception and representation of a person is of space is also important. Therefore, the level of human satisfaction from the environment is related to processes of social comparison and cultural differences (Fergas, 2000).

Undoubtedly, the public realm is the place where the greatest amount of contact and interaction occurs within human beings. Basically, the public space emphasizes unrestricted access to diverse activities, the most notable of which is social interaction. In other words, the public space is a place, the place of simultaneous activity, the place for the presentation, the discovery of differences and identities, an area for recognition. The place where an individual acquires knowledge of the differences between himself and others (Astana, 2011; p. 70-71). One of the most important dimensions of public spaces is the creation of opportunities for social interaction. In these interactions, people establish a stronger relationship with their environment and society, and their sense of belonging and collective identity are reinforced, with the formation of social networks and the increase of collective participation. In the public space, face-to-face communication, dialogue, friendship, mutual interest, participation in social activities and sense of co-operation are created. The motive for gathering can be different, from an intimate dialogue from two to a larger general scale, including customs, rituals, shows, celebrations, sporting events, etc. (Grasshopper \& Gypsum, 2011; 2). People come to social spaces to feel other people (Bentley, 2003; p. 185). The opportunity to meet others is a prerequisite for promoting unexpected and informal social interaction. Headquarters and places of behavior should be placed in places of public acceptance so that people will tend to be present in them. Undoubtedly, it is difficult to create a room that encourages public life in today's society. But in fact, it is much more likely than what is happening. The problem is merely the art of inviting people to come, stay, and use space, until they are more or less unintentionally led to public spaces that are not inviting (Gol, 2007; p. 52).

7. Nature Perception

In many human environments, paying attention to the natural environment has been the focus of a beautiful landscape supplier. Although newer perspectives emphasize the necessity of expanding this approach comprehensively. "Our ability to understand the quality of nature, as in art with beautiful components, begins, and then the sequence of sequences from the beautiful to the values that so far the language is unable to express them (Bell, 2003; p. 108)". Many of the architects' encounters with nature are limited to nature, and nature has attracted attention from the provider of and beauty. But the attitude to nature from the angle of perception of the environment implies a more comprehensive approach, which involves direct perception and understanding to implicit meanings and psychological and well-being values arising from interaction with the environment and natural elements, and perceptual feedback in the system of user activities and behaviors The environment (Daneshvar Moghaddam, Bahrain \& Einifar, 2011; p. 30).

Exploring the literature of the subject, centered on how to perceive nature in the environment, implies three general approaches that are identical in terms of expression. The first approach considers the perception of nature, including sensory perception and mental perception. Sensory perception of visual aesthetics and mental perception of symbolic aesthetics, symbolic meanings and implied meanings in relation to place. The second approach considers the perception of nature in the environment, including three aspects of direct perception, indirect perception and symbolic perception (2008, Kellert). In this regard, direct perception of the direct presence of natural elements in the environment implies indirect perception of historical and cultural aspects such as associations in the environment and symbolic perception of the transcendental interactions of man and natural elements in the environment.

8. The effect of nature perception on the social space of the public space

Nature's perception of the physical environment has a collective image. Sociability is influenced by several factors that the ability to interact with the environment and natural elements are among the set of components that characterize this quality. The characteristics and of spaces in relation to the natural context, the presence of natural elements in space, natural perspectives, direct perception of events is affected by the balance of the physical variables of the environment such as temperature, natural ventilation, etc. If the environment makes it possible to perceive nature elements in space, the perception of nature has a significant effect on the sociability of space, which causes the concentration of space and the formation of activity centers in a certain context of activity (Daneshvar Moghaddam, Bahrain and Einifar, 2011; p. 34-33).

\section{Conclusions}

Sustainability thoughts and social interactions have been the main focus of attention for most experts for many years. Recent sustainability perspectives also address social and economic issues in addition to environmental issues. Hence, the cultural and social influences of the artificial environment are one of the most effective factors in sustainability. Considering the need of the elderly to have social interactions, considering the specific psychological conditions of this period, by planning and designing the public spaces of their residential complexes attempts to impress these spaces. Creating social characteristics in these public spaces increases the possibility of social encounters by creating a willingness to re-enter these spaces in the users. Nature perception 
is one of the factors affecting the sociability of space, and an environment that can provide perceptual perceptions of nature, both visually and physically, have a significant effect on the formation of social interactions. This could be achieved by designing better environments in elderly residential complexes to prevent their isolation and provide better quality for the public spaces of these collections.

\section{References}

1. Rafizadeh, Neda Engineer, Autumn and Winter (2003), Designing an Appropriate Architecture for the Elderly, Journal of Architecture and Urban Research, no. 37, p. 112.

2. Pour Jafar, Mohammad Reza, Daghahi, Ali Akbar, Bomanian, Mohammad Reza, Sadeghi, Alireza and Ahmadi, Faryal (2010). Providing imaginative environments that affect the formation of public spaces. Promoting good aging. Elderly Journal, Vol. 5, No. 15, Tehran: 22 - 34

3. Herawi Karimavi, Majid, Paradeh, Majid and Nahid, Jalali, Amir and Pishmandazi, Zahra (2003). The study of psychological needs of elderly people in Tehran. Nursing Daneshvar, Volume 11, Number 48, Tehran: 59-66

4. Pasha, Gholamreza, Safarzadeh, Sahar and Mashak, Roya (2007). Comparison of general health and social support among the elderly living in the elderly and elderly homes in the family. Quarterly Journal of Powhide Family, Third Year, No. 9. Tehran: 517-503

5. Rafizadeh, Neda (2001) "Day Care Centers, Design Guidelines for the Nursing Home Architecture", Factual Report, Issue Number 338, Tehran, Ministry of Housing and Urban Development.

6. Bagheri Lankarani, Narges (2003) "The Elder from One's View", elderly journal, No. 4.

7. Shamloo, Gholam Ali (1364) "What is old age, why are we getting old?" Tehran, Chahar.

8. Proceedings of the Seniority (2000) "Psychological Issues, Exercise and Nutrition in the Elderly", Tehran, Nichakar Women's Group.

9. Sturant, Martha (1998) "Psychology of Aging", Tehran, Health Organization.

10. Dreiber, Inspiration (1364) "Continuation of neglect" Journal of Transportation Industry, No. 4, Spring.

11. Tabatabai, Fakhrasadat, Aminzadeh, Rahim (1392). Investigation of Sustainability Components in Zandieh Architecture. National Conference on Architecture, Urban Development and Sustainable Development, Mashhad, Khavaran Higher Education Institute.

12. My Left, David (1386). Creation of neighborhoods and places in the human environment. Translation by Manouchehr Tabibian, Tehran University Press, Tehran.

13. Seedlings, Kaveh, Gypsum, Maryam (1390). Investigating the role of public spaces in the formation of social interactions in order to create social sustainability in cities. National Conference on Civil Engineering, Architecture, Urban Planning and Energy Management. Ardestan Islamic Azad University, Ardestan Branch.

14. Dignity, Seyyed Hussein (1391). From Style to Identity in Architecture. Quarterly Journal of Fine Arts, Volume 17, Number 2, Tehran: 65-74

15. Davoudi, Saeed, Fallah, Mohammad Hassan and Ali Abadi, Mohammad (1392). Sustainability and Architecture, Determining the Indicators Influencing the Development of Sustainability in Architecture. National Conference on Architecture and Urban Development and Sustainable Development, Focusing on Native Architecture to the Sustainable City, Mashhad, Khavaran Higher Education Institute.

16. Daneshpour, Seyyed Abdul Hadi, Guardian, Maryam (2007). Public spaces and factors affecting collective life. Journal of Gardening Science, Volume 4, Issue 7, Tehran, 19-28.

17. Sefi Divine, Seyfollah. (2002), Sociology of Social Issues in Iran (Proceedings and Opinions). Tehran: Publication of Sina Pajohan Sina.

18. Taywood, Julia. (2000), Interpersonal Communication: Psychology of Social Interactions. (Mehrdad Firozbakht, Translator). Tehran: Mahtab Publication.

19. Alexander, Christopher. (2008), Pattern Language: Cities, (Reza Karbala'i Nouri, Translator). Tehran: Center for Research and Development of Urbanism and Architecture

20. Sanctuary, Mohammad Hussein, Enemy Gharin, Parinas, Dehdari, Tahereh and Dashman Gher, Leyla (2012). Factors Affecting a Healthy Lifestyle from the Viewpoint of the Elderly. Eldest Quarterly, Seventh Edition, No. 27, Tehran: 47-58

21. Hashemzadeh, Hashem, Asadi Boalourdi, Samira (1388). Use of daily living space to improve the quality of life of the elderly. Two Utopian Architectural and Urban Planning Quarterly, Second Edition, No. 2, Tehran: 75-90.

22. Fergus, Joseph P. (2000), Psychology of Social Interaction, Interpersonal Behavior, Translation by Mehrdad Firouzbakht and Kheshayar Beigi, Tehran: Mahd Publication.

23. Lang, John (2002), Creation of Architectural Theory, The Role of Behavioral Sciences in Environmental Design, Tehran University Press and Publishing Institute, Spring Edition.

24. Astana, Lily (1390). Man, space and social interactions. Daneshvar Newspaper, Volume Twenty, No. 198 - 
199. Isfahan: 66-72.

25. Bentley, Ein and others (1382). Responsive environments. Translation by Mostafa Behzadfar, Science \& Technology University Press: Tehran.

26. Flowers, Jan (1386). Life, spaces, buildings. Two Iranian Journal of Architecture, Vol. 8, No. 29-30, Tehran: 48-56

27. Bell, Simon (1382). Parallax, pattern, perception, process. Translated by Behnaz Aminzadeh, First Edition, Tehran University Press, Tehran.

28. Daneshvar Moghadam, Gholkhah, Bahrain, Seyyed Hossein and Einifar, Alireza (2011). The analysis of the social nature of the physical environment is influenced by the perception of nature in the human environment. Beautiful Arts, No. 45, Tehran: 25 -36.

29. Moustakas, C., (1961). Loneliness, Prentice Hall Inc: NEW YORK

30. Shearer R, Davidhizar, R., (1999). Conquering loneliness, Elder Care, 11 (2): 12-15.

31. Gifford R. (1997) environmentL Psychology, Allyn and bacon, Boston.

32. skjaeveland, O and garling t. (1997) effects of interactional space on neighbouring, journal of Environmental Psychology, 17, 181-199.

33. Kallert, Stephen R. (2005). Building for Life, ISLANDPRESS: Washington. 